\title{
Reduction of Intensity Noise in Semiconductor Lasers by Simultaneous Usage of the Superposition of High Frequency Current and the Electric Negative Feedback
}

\author{
Minoru YAMADA $^{\dagger}$, Member, $_{\text {Itaru TERA }}^{\dagger \text { a) }}$, Kenjiro MATSUOKA ${ }^{\dagger}$, Takuya HAMA $^{\dagger}$, Nonmembers, $^{\dagger}$ \\ and Yuji KUWAMURA ${ }^{\dagger}$, Member
}

\begin{abstract}
SUMMARY Reduction of the intensity noise in semiconductor lasers is an important subject for the higher performance of an application. Simultaneous usage of the superposition of high frequency current and the electric negative feedback loop was proposed to suppress the noise for the higher power operation of semiconductor lasers. Effective noise reduction of more than $25 \mathrm{~dB}$ with $80 \mathrm{~mW}$ operation was experimentally demonstrated.

key words: semiconductor laser, optical feedback noise, noise reduction, electric negative feedback, high frequency current superposition
\end{abstract}

\section{Introduction}

Semiconductor lasers are used as light sources in optical communication, optical disc and optical measuring systems. The semiconductor lasers tend to generate excess intensity noise, so-called the optical feedback noise, which is induced by reinjection of the output light into the laser by reflection at the surface of connecting optical devices, as well as the quantum noise which is caused by intrinsic fluctuations on the electron and photon numbers [1], [2]. The optical feedback noise can be suppressed by superposition of the HF (High Frequency) current [3] or by utilization of the selfpulsation laser [4]. However, in some cases the noise can not reduce sufficiently only by these reduction methods [5], [6].

On the other hand, an operating method of the lasers setting an electric negative feedback loop from the photodetector to the driving current was proposed for the noise reduction [7], [8]. Furthermore, another operating method with the electric positive and negative feedback (EPNF) has also been proposed, where an electric positive feedback is applied with a high frequency to induce electrical oscillation giving similar effect with the superposition of HF current or the self-pulsation laser, while the electric negative feedback loop is applied in the low frequency region simultaneously [9]. The EPNF method is achieved with a single electronic amplifier by utilizing the characteristic that phase delay of an electric signal in the low frequency region is almost constant but increases rapidly around at the cut off frequency.

\footnotetext{
Manuscript received December 28, 2011.

Manuscript revised March 12, 2012.

${ }^{\dagger}$ The authors are with Optical Communication Laboratory, Division of Electrical Engineering and Computer Science, Graduate School of Natural Science and Technology, Kanazawa University, Kanazawa-shi, 920-1192 Japan.

a)E-mail: tera@popto5.ec.t.kanazawa-u.ac.jp

DOI: 10.1587/transele.E95.C.1444
}

Not only the optical feedback noise but also the quantum noise can be suppressed effectively by the EPNF method [9].

The noise reduction method should be applicable for the wider operating ranges of the optical power and the signal frequency. However, application of the EPNF method to the high power semiconductor laser is not easy, because preparation of an idealized electronic amplifier, which can operate with very large power together with extremely low noise level, is required. The higher power electronic amplifier gives the higher noise with the narrower frequency band-width, in general.

In this letter, we propose a new noise reduction method by simultaneous usage of the superposition of HF current and the electric negative feedback loop. This method is aimed to extend the operation to the higher optical power and the wider frequency range including the DC region. Amount of the HF current and loop gain of the electric negative feedback can be optimised for the more effective noise reduction in this new method. The electric negative feedback loop covering the DC and the AC ranges can work also as the auto power control (APC) system.

\section{Experimental Scheme}

Conceptual illustration for simultaneous usage of the superposition of HF current and the electric negative feedback loop is shown in Fig. 1. One part of the output light from the front facet is detected by a photo-detector, and is put in an electrical feedback loop with an electronic amplifier to the

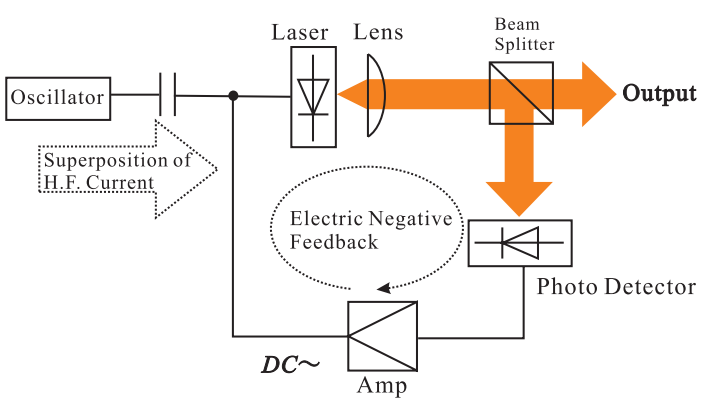

Fig. 1 Conceptual illustration for simultaneous usage of the superposition of HF current and the electric negative feedback loop. 


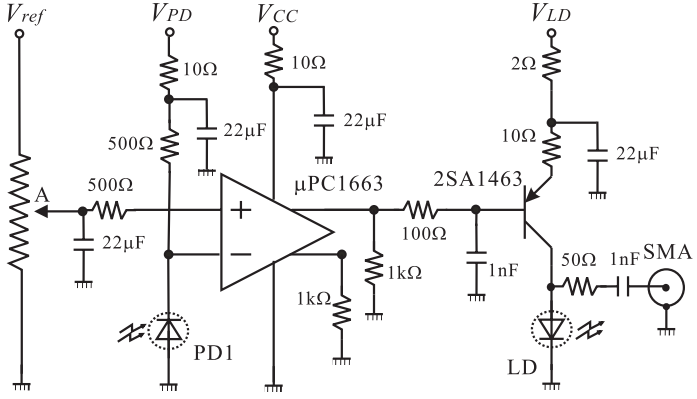

Fig. 2 Driving circuit for simultaneous usage of the superposition of HF current and the electric negative feedback loop.

driving current source as a negative feedback including. The effect of this negative feedback loop is almost same as the conventional auto power control (APC) system. However, a remarking feature of our method is that we need to detect the optical output from the front facet not from the back facet to perform noise reduction. In the conventional APC system, the light emitted from the back facet is utilized, because the detector can be set at close position to the laser in a mounting cap. However, fluctuation of the light from the front facet and that from the back facet is not identical [8]. Phenomena of the different fluctuations may be caused by slightly non-uniform structure of the active region or asymmetric reflectivities of the two end facets. Then, the light emitted from the back facet is not suitable for application of the noise reduction.

The HF current for the superposition is generated in an external oscillator, and put into the semiconductor laser.

An assembled driving circuit for simultaneous usage of the superposition of HF current and the electric negative feedback loop is shown in Fig. 2. An operational amplifier, $\mu \mathrm{PC} 1663$, and a transistor, 2SA1463, are used for the amplification of the intensity noise, and controlling the driving current. DC driving current is controlled by adjusting the variable register which is indicated with A in Fig. 2. PD1 is a photo-detector and LD is a semiconductor laser. The PD1 detects one part of the LD output, and the circuit is designed to control DC driving current to a fixed value and suppress the noise by the negative feedback. When the electric negative feedback is not required, no light is put into the PD1, and value of the variable register is readjusted to drive the LD with same output power.

The HF current is brought in the LD though a SMA connector from an external oscillator.

Experimental set up is shown in Fig. 3. Used laser was a high power $\mathrm{AlGaAs}$ semiconductor laser whose lasing wavelength is $\lambda=830 \mathrm{~nm}$, threshold current is $53.5 \mathrm{~mA}$, and slope efficiency is $0.93 \mathrm{~W} / \mathrm{A}$ when temperature of the laser is $T=25^{\circ} \mathrm{C}$. The light output from the laser was reflected by a mirror and injected into the laser. Distance between the laser and the mirror is $\ell=15 \mathrm{~cm}$. Optical feedback ratio $\Gamma$ was changed by an attenuator (ATT) and monitored by a power-meter through a beam splitter and a photo-detector PD2.

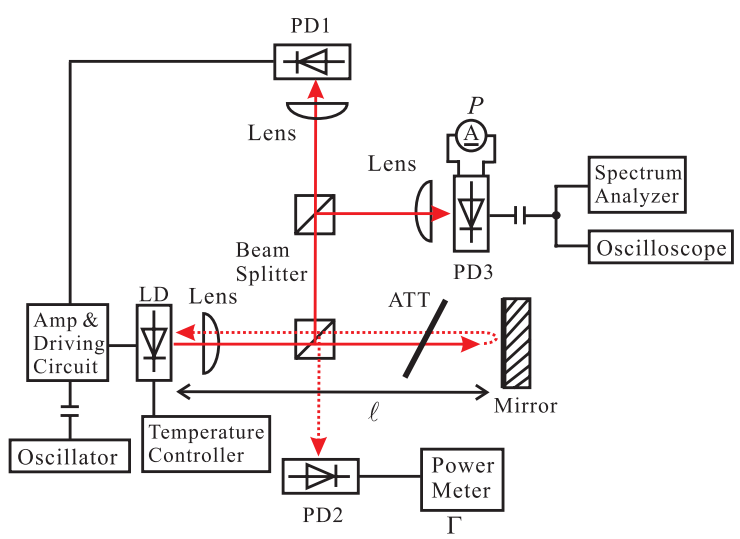

Fig. 3 Experimental set up.

The light output was detected by PD1 passing through two beam splitters and was put into the driving circuit shown in Fig. 2. Electric amplification ratio from the PD1 to the LD was $74 \mathrm{~dB}$ in the frequency range from $\mathrm{DC}$ to several $\mathrm{MHz}$.

DC power and the intensity noise of the light output were measured by another photo-detector PD3 with a spectrum analyzer or an oscilloscope.

\section{Experimental Results}

We measured the intensity noise of the semiconductor laser under following conditions : 1) Free running operation without the optical feedback, by which the quantum noise level is determined, 2) operation under optical feedback tuned to give the highest noise level without any noise suppression technique, 3) operation under optical feedback with the superposition of HF current, 4) operation under optical feedback with the electric negative feedback, and 5) operation under optical feedback with both the superposition of HF current and the negative feedback.

Frequency variation of the noise is shown in Fig. 4. The output power of the laser $P$ was $32 \mathrm{~mW}$ and the optical feedback ratio was $\Gamma=0.002$. The line with lozenges was measured without the optical feedback as a free running operation and indicates the quantum noise level. Other lines are with the optical feedback tuned, to give the highest noise level. Actually, the noise was increased more than $35 \mathrm{~dB}$ at $100 \mathrm{kHz}$ by the optical feedback. However, the noise was reduced $22 \mathrm{~dB}$ by help of either the superposition HF current or the electric negative feedback. The noise was more reduced $37 \mathrm{~dB}$ by simultaneous application of the superposition HF current and the electric negative feedback. The reduced noise level is below the quantum noise.

Variation of the noise with output power $P$ is shown in Fig. 5. Measured frequency of the noise was $200 \mathrm{kHz}$. The electric negative feedback can suppress the noise more effectively than the superposition of HF current and suppress the noise lower level than the quantum noise in some conditions. However, the simultaneous usage of the two methods can suppress the noise much more. Although the suppression effect reduces for the higher power, $25 \mathrm{~dB}$ reduction 


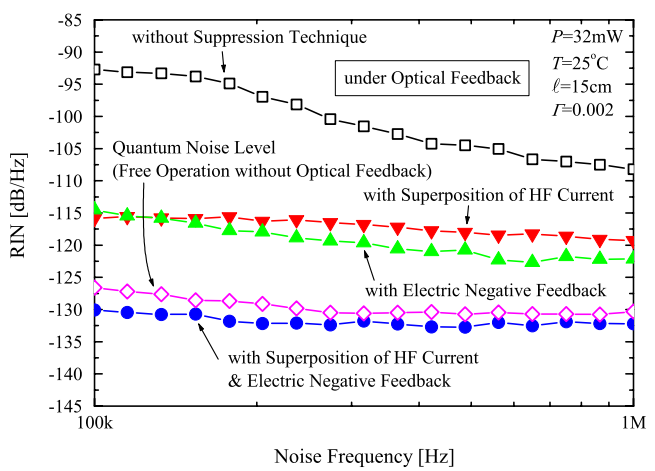

Fig. 4 RIN vs. noise frequency.

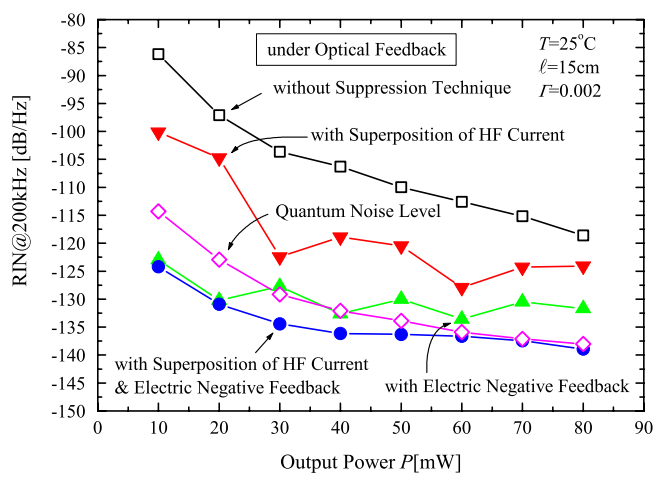

Fig. 5 RIN vs. Output Power $P$.

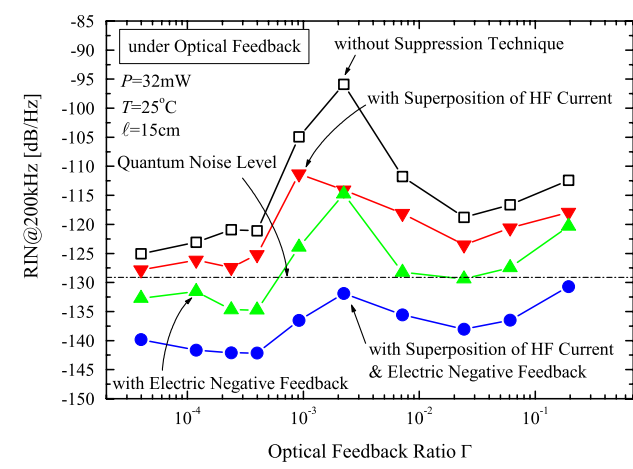

Fig. 6 RIN vs. optical feedback ratio $\Gamma$.

was confirmed at $80 \mathrm{~mW}$ operation.

Variation of the noise with optical feedback ratio $\Gamma$ is shown in Fig. 6. The chain line is the quantum noise level. The noise becomes the largest at $\Gamma \approx 2 \times 10^{-3}$, which must be caused by the mode competition phenomena among the lasing mode of the laser cavity [5], [8]. The highest noise could not be suppressed only either by the superposition of HF current or by the electric negative feedback, but was well suppressed by the simultaneous usage of the two methods down to a lower level than the quantum noise.

\section{Conclusion}

As an excellent noise reduction scheme, simultaneous usage of the superposition of HF current and the electronic negative feedback is proposed. Effective noise reduction of more than $25 \mathrm{~dB}$ with $80 \mathrm{~mW}$ operation was experimentally demonstrated. This reduction scheme can suppress not only the optical feedback noise but also the quantum noise, and is applicable to the higher power laser with the wider frequency range including DC region.

\section{References}

[1] T. Morikawa, Y. Mituhasi, J. Shimada, and Y. Kajima, "Return-beaminduced oscillations in self-coupled semiconductor lasers," Electron. Lett., vol.12, pp.435-436, July 1976.

[2] I. Ikusima and M. Maeda, "Self-coupled phenomena of semiconductor lasers caused by an optical fiber," IEEE J. Quantum Electron. vol.QE-14, no.5, pp.331-332, May 1978.

[3] A. Ohishi, M. Chinone, M. Ojima, and A. Arimoto, "Noise characteristics of high-frequency superposed laser diodes for optical disc systems," Electron Lett., vol.20, pp.821-822, Jan. 1984.

[4] S. Matsui, H. Takiguchi, H. Hayashi, S. Yamamoto, S. Yano, and T. Hijikata, "Suppression of feedback-induced noise in short-cavity V-channeled suvstrate inner stripe lasers with self-oscillation," Appl. Phys. Lett., vol.43, pp.219-221, Aug. 1983.

[5] M. Yamada, S. Yamamura, and T. Okamoto, "Characterization of the feedback induced noise in semiconductor laser under superposition of high frequency current," IEICE Trans. Electron., vol.E84-C, no.10, pp.1588-1596, Oct. 2001.

[6] M. Yamada, Y. Ishikawa, S. Yamamura, M. Kidu, A. Kanamori, and Y. Aoki, "Experimental characterization of the feedback induced noise in self-pulsing lasers," IEICE Trans. Electron., vol.E82C, no.12, pp.2241-2247, Dec. 1999.

[7] M. Nakamura, T. Tamura, and T. Ozeki, "Elimination of laser noise and distortion induced by reflected wave," Tech. Dig. 3rd Int. Conf. IOOC, Washington, DC, 1981.

[8] M. Yamada, N. Nakaya, and M. Funaki, "Characteristics of modehopping noise and its suppression with the help of electric negative feedback in semiconductor lasers," IEEE J. Quantum Electron., vol.QE-23, no.8, pp.1297-1302, Aug. 1987.

[9] M. Yamada, M. Huda, E. Teraoka, and Y. Kuwamura, "Effective noise reduction by electric positive and negative feedback in semiconductor lasers," IEEE J. Quantum Electron., vol.45, no.10, pp.1248-1254, Oct. 2009. 